\title{
Investigation of solvability condition for sixth-order boundary value problem
}

\author{
Akram Hassan Mahmood \\ Department of Mathematics \\ College of Education \\ University of Mosul
}

\author{
Alaa Ahmed Mohamed \\ M.SC. of Mathematics
}

\begin{abstract}
Received
26 / 09 / 2010

Accepted

05 / 01 / 2011

الملخص

تتاولنا في هذا البحث دراسة شرط الحل لمعادلة تفاضلية خطية غير متجانسة من الرتبة

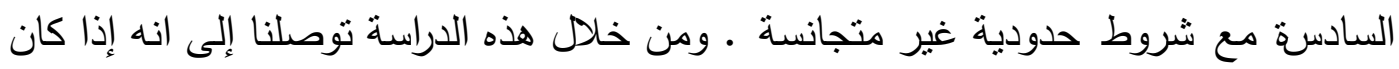

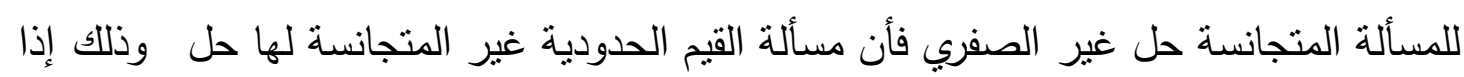

حقق الحد غير المتجانس شرط الحل وقد تم تأكيد النتائج من خلال المثال التوضيحي المعطى.
\end{abstract}

\begin{abstract}
This paper is concerned with the solvability condition for nonhomogenous linear boundary value problem for sixth-order ordinary differential equation.

Throughout this study, we observed that, when the homogenous problem

have nontrivial solution,then the nonhomogenous boundary value problem have a solution in case of nonhomogenous term that satisfied the solvability condition.

We justified our results through the given example.

Keywords :Sixth-order boundary value problem, self-adjoint problem.

\section{(1)- Introduction}

There is a relationship between homogenous and nonhomogenous linear boundary value problem as there is between homogenous and nonhomogenous linear algebra system. A nonhomogenous boundary value problem has a unique solution and the corresponding homogenous problem has only the trivial solution, then a nonhomogenous problem has either no solution or infinity many, and the corresponding homogenous
\end{abstract}


problem has nontrivial solution Boyce [1]. The solvability condition is derived for the case of fourth -order nonhomogenous boundary value problem Nayfeh [4]. In Mahmood et al [3] and Mahmood [2], the solvability condition for certain eigenvalue problem by perturbation method was studies .In both Noor et al [5] and Shen [6] applied the homotopy perturbation method for solving fourth -order boundary value problems and fifth-order boundary value problems.

This paper deals with investigation of the solvability for the following boundary value problem

$$
\begin{aligned}
& p_{6}(x) \varphi^{V I}+p_{5}(x) \varphi^{V}+p_{4}(x) \varphi^{I V}+p_{3}(x) \varphi^{\prime \prime \prime}+p_{2}(x) \varphi^{\prime \prime}+p_{1}(x) \varphi^{\prime}+ \\
& +p_{0}(x) \varphi=f(x) \\
& \varphi(a)=\beta_{1}, \varphi^{\prime}(a)=\beta_{2}, \varphi^{\prime \prime}(a)=\beta_{3}, \varphi(b)=\beta_{4}, \varphi^{\prime}(b)=\beta_{5}, \varphi^{\prime \prime}(b)=\beta_{6}
\end{aligned}
$$

Where $P_{i}(x) \in c^{i}[a, b], i=1,2,3,4,5,6, \quad P_{6}(x) \neq 0 \quad$ in the interval [a,b] and $f(x)$ is continuous function in the same interval and also $\beta_{1}, \beta_{2}, \beta_{3}, \beta_{4}, \beta_{5}$ and $\beta_{6}$ are real constants.

\section{(2)- Solvability Condition for the problem}

In this section, we will try to give a theorem which is the main basis of solvability condition for the problem (1.1). It is worth noting that when the homogenous have a nontrivial solution, the nonhomogenous equations have a solution if and only if the nonhomogenous parts satisfy a solvability condition [4].

\section{Theorem}

The desired solvability condition that the problem (1.1-1.2) has a solution is

$$
\begin{aligned}
& {\left[P_{6} u^{\prime \prime \prime} \beta_{6}+\left(4 P_{6}^{\prime} u^{\prime \prime \prime}+P_{6} u^{I V}-P_{5} u^{\prime \prime \prime}\right) \beta_{5}-\left(10 P_{6}^{\prime \prime} u^{\prime \prime \prime}+5 P_{6}^{\prime} u^{I V}+P_{6} u^{V}\right.\right.} \\
& \left.\left.-4 P_{5}^{\prime} u^{\prime \prime \prime}-P_{5} u^{I V}+P_{4} u^{\prime \prime \prime}\right) \beta_{4}\right]_{b}-\left[P_{6} u^{\prime \prime \prime} \beta_{3}+\left(4 P_{6}^{\prime} u^{\prime \prime \prime}+P_{6} u^{I V}-P_{5} u^{\prime \prime \prime}\right) \beta_{2}\right. \\
& \left.-\left(10 P_{6}^{\prime \prime} u^{\prime \prime \prime}+5 P_{6}^{\prime} u^{I V}+P_{6} u^{V}-4 P_{5}^{\prime} u^{\prime \prime \prime}-P_{5} u^{I V}+P_{4} u^{\prime \prime \prime}\right) \beta_{1}\right]_{a}=\int_{a}^{b} f(x) u(x) d x
\end{aligned}
$$

\section{Proof}

To determine the solvability condition for the problem (1.1-1.2) we multiply (1.1) by $u(x)$ and integrate it from $x=a$ to $x=b$, we obtain

$$
\begin{gathered}
\int_{a}^{b} P_{6} u \varphi^{V I} d x+\int_{a}^{b} P_{5} u \varphi^{V} d x+\int_{a}^{b} P_{4} u \varphi^{I V} d x+\int_{a}^{b} P_{3} u \varphi^{\prime \prime \prime} d x+\int_{a}^{b} P_{2} u \varphi^{\prime \prime} d x+ \\
+\int_{a}^{b} P_{1} u \varphi^{\prime} d x+\int_{a}^{b} P_{0} u \varphi d x=\int_{a}^{b} u(x) f(x) d x
\end{gathered}
$$


We integrate by parts the integrals in (2.2) to transfer the derivatives from $\varphi$ to $u$, we note that

$$
\begin{gathered}
\int_{a}^{b} P_{6} u \varphi^{V I} d x=\left.\left(P_{6} u\right) \varphi^{V}\right|_{a} ^{b}-\int_{a}^{b}\left(P_{6} u\right)^{\prime} \varphi^{V} d x=\left[P_{6} u \varphi^{V}-\left.\left(P_{6} u\right)^{\prime} \varphi^{I V}\right|_{a} ^{b}\right. \\
+\int_{a}^{b}\left(P_{6} u\right)^{\prime \prime} \varphi^{I V} d x=\left[P_{6} u \varphi^{V}-\left(P_{6} u\right)^{\prime} \varphi^{I V}+\left.\left(P_{6} u\right)^{\prime \prime} \varphi^{\prime \prime \prime}\right|_{a} ^{b}\right. \\
-\int_{a}^{b}\left(P_{6} u\right)^{\prime \prime \prime} \varphi^{\prime \prime \prime} d x=\left[P_{6} u \varphi^{V}-\left(P_{6} u\right)^{\prime} \varphi^{I V}+\left(P_{6} u\right)^{\prime \prime} \varphi^{\prime \prime \prime}-\left.\left(P_{6} u\right)^{\prime \prime \prime} \varphi^{\prime \prime}\right|_{a} ^{b}\right. \\
+\int_{a}^{b}\left(P_{6} u\right)^{I V} \varphi^{\prime \prime} d x=\left[P_{6} u \varphi^{V}-\left(P_{6} u\right)^{\prime} \varphi^{I V}+\left(P_{6} u\right)^{\prime \prime} \varphi^{\prime \prime \prime}-\left(P_{6} u\right)^{\prime \prime \prime} \varphi^{\prime \prime}+\left.\left(P_{6} u\right)^{I V} \varphi^{\prime}\right|_{a}\right. \\
-\int_{a}^{b}\left(P_{6} u\right)^{V} \varphi^{\prime} d x=\left[P_{6} u \varphi^{V}-\left(P_{6} u\right)^{\prime} \varphi^{I V}+\left(P_{6} u\right)^{\prime \prime} \varphi^{\prime \prime \prime}-\left(P_{6} u\right)^{\prime \prime \prime} \varphi^{\prime \prime}+\left(P_{6} u\right)^{I V} \varphi^{\prime}\right. \\
-\left(P_{6} u\right)^{V} \varphi_{a}^{b}+\int_{a}^{b}\left(P_{6} u\right)^{V I} \varphi d x
\end{gathered}
$$

In similar way

$$
\begin{aligned}
& \int_{a}^{b} P_{5} u \varphi^{V} d x=\left[P_{5} u \varphi^{I V}-\left(P_{5} u\right)^{\prime} \varphi^{\prime \prime \prime}+\left(P_{5} u\right)^{\prime \prime} \varphi^{\prime \prime}-\left(P_{5} u\right)^{\prime \prime \prime} \varphi^{\prime}+\left.\left(P_{5} u\right)^{I V} \varphi\right|_{a} ^{b}-\int_{a}^{b}\left(P_{5} u\right)^{V} \varphi d x,\right. \\
& \int_{a}^{b} P_{4} u \varphi^{I V} d x=\left[P_{4} u \varphi^{\prime \prime \prime}-\left(P_{4} u\right)^{\prime} \varphi^{\prime \prime}+\left(P_{4} u\right)^{\prime \prime} \varphi^{\prime}-\left.\left(P_{4} u\right)^{\prime \prime \prime} \varphi\right|_{a} ^{b}+\int_{a}^{b}\left(P_{4} u\right)^{I V} \varphi d x,\right. \\
& \int_{a}^{b} P_{3} u \varphi^{\prime \prime \prime} d x=\left[P_{3} u \varphi^{\prime \prime}-\left(P_{3} u\right)^{\prime} \varphi^{\prime}+\left.\left(P_{3} u\right)^{\prime \prime} \varphi\right|_{a} ^{b}-\int_{a}^{b}\left(P_{3} u\right)^{\prime \prime \prime} \varphi d x,\right. \\
& \int_{a}^{b} P_{2} u \varphi^{\prime \prime} d x=\left[P_{2} u \varphi^{\prime}-\left.\left(P_{2} u\right)^{\prime} \varphi\right|_{a} ^{b}+\int_{a}^{b}\left(P_{2} u\right)^{\prime \prime} \varphi d x,\right. \\
& \int_{a}^{b} P_{1} u \varphi^{\prime} d x=\left[\left.P_{1} u \varphi\right|_{a} ^{b}-\int_{a}^{b}\left(P_{1} u\right)^{\prime} \varphi d x\right.
\end{aligned}
$$

Therefore we can rewrite (2.2) as

$$
\begin{aligned}
& \int_{a}^{b} \varphi\left[\left(P_{6} u\right)^{V I}-\left(P_{5} u\right)^{V}+\left(P_{4} u\right)^{I V}-\left(P_{3} u\right)^{\prime \prime \prime}+\left(P_{2} u\right)^{\prime \prime}-\left(P_{1} u\right)^{\prime}+\left(P_{0} u\right)\right] d x \\
& +\left(\left(P_{6} u\right) \varphi^{V}-\left(\left(P_{6} u\right)^{\prime}-\left(P_{5} u\right)\right) \varphi^{I V}+\left(\left(P_{6} u\right)^{\prime \prime}-\left(P_{5} u\right)^{\prime}+\left(P_{4} u\right)\right) \varphi^{\prime \prime \prime}\right. \\
& -\left(\left(P_{6} u\right)^{\prime \prime \prime}-\left(P_{5} u\right)^{\prime \prime}+\left(P_{4} u\right)^{\prime}-\left(P_{3} u\right)\right) \varphi^{\prime \prime}+\left(\left(P_{6} u\right)^{I V}-\left(P_{5} u\right)^{\prime \prime \prime}+\left(P_{4} u\right)^{\prime \prime}\right. \\
& \left.-\left(P_{3} u\right)^{\prime}+\left(P_{2} u\right)\right) \varphi^{\prime}-\left(\left(P_{6} u\right)^{V}-\left(P_{5} u\right)^{I V}+\left(P_{4} u\right)^{\prime \prime \prime}-\left(P_{3} u\right)^{\prime \prime}+\left(P_{2} u\right)^{\prime}\right. \\
& \left.\left.-\left(P_{1} u\right)\right) \varphi\right\}_{a}^{b}=\int_{a}^{b} f(x) u(x) d x
\end{aligned}
$$

To find the differential equation describing the adjoint $u$, we set the coefficient of $\varphi$ in the integral on the left -hand side of (2.3) equal zero, we have: 
$\left(P_{6} u\right)^{V I}-\left(P_{5} u\right)^{V}+\left(P_{4} u\right)^{V}-\left(P_{3} u\right)^{\prime \prime \prime}+\left(P_{2} u\right)^{\prime \prime}-\left(P_{1} u\right)^{\prime}+\left(P_{0} u\right)=0$

which is the adjoint homogenous differential equation corresponding to (1.1). In order that the homogenous differential equation (1.1) be self adjoint, (2.4) must be the same as the homogenous equation (1.1).

Expanding the derivatives in (2.4) and obtain

$$
\begin{aligned}
& P_{6} u^{V I}+\left(6 P_{6}^{\prime}-P_{5}\right) u^{V}+\left(15 P_{6}^{\prime \prime}-5 P_{5}^{\prime}+P_{4}\right) u^{I V}+\left(20 P_{6}^{\prime \prime \prime}-10 P_{5}^{\prime \prime}+4 P_{4}^{\prime}\right. \\
& \left.\quad-P_{3}\right) u^{\prime \prime \prime}+\left(15 P_{6}^{I V}-10 P_{5}^{\prime \prime \prime}+6 P_{4}^{\prime \prime}-3 P_{3}^{\prime}+P_{2}\right) u^{\prime \prime}+\left(6 P_{6}^{V}-5 P_{5}^{I V}+4 P_{4}^{\prime \prime \prime}\right. \\
& \left.-3 P_{3}^{\prime \prime}+2 P_{2}^{\prime}-P_{1}\right) u^{\prime}+\left(P_{6}^{V I}-P_{5}^{V}+P_{4}^{I V}-P_{3}^{\prime \prime \prime}+P_{2}^{\prime \prime}-P_{1}^{\prime}+P_{0}\right) u=0
\end{aligned}
$$

Comparing (2.5) with (1.1) we obtain

$$
\begin{aligned}
& P_{5}=6 P_{6}^{\prime}-P_{5} \\
& P_{4}=15 P_{6}^{\prime \prime}-5 P_{5}^{\prime}+P_{4} \\
& P_{3}=20 P_{6}^{\prime \prime \prime}-10 P_{5}^{\prime \prime}+4 P_{4}^{\prime}-P_{3} \\
& P_{2}=15 P_{6}^{I V}-10 P_{5}^{\prime \prime \prime}+6 P_{4}^{\prime \prime}-3 P_{3}^{\prime}+P_{2} \\
& P_{1}=6 P_{6}^{V}-5 P_{5}^{I V}+4 P_{4}^{\prime \prime \prime}-3 P_{3}^{\prime \prime}+2 P_{2}^{\prime}-P_{1} \\
& P_{0}=P_{6}^{V I}-P_{5}^{V}+P_{4}^{I V}-P_{3}^{\prime \prime \prime}+P_{2}^{\prime \prime}-P_{1}^{\prime}+P_{0}
\end{aligned}
$$

or

$$
P_{5}=3 P_{6}^{\prime} \quad, \quad P_{3}=-5 P_{6}^{\prime \prime \prime}+2 P_{4}^{\prime} \quad, \quad P_{1}=3 P_{6}^{V}-P_{4}^{\prime \prime \prime}+P_{2}^{\prime}
$$

Then (1.1) becomes

$$
P_{6} \varphi^{V I}+3 P_{6}^{\prime} \varphi^{V}+P_{4} \varphi^{I V}+\left(2 P_{4}^{\prime} u-5 P_{6}^{\prime \prime \prime}\right) \varphi^{\prime \prime \prime}+P_{2} \varphi^{\prime \prime}+\left(3 P_{6}^{V}-P_{4}^{\prime \prime \prime}+P_{2}^{\prime}\right) \varphi^{\prime}+P_{0} \varphi=0
$$

Which can be written as

$$
\frac{d^{3}}{d x^{3}}\left(P_{6} \varphi^{\prime \prime \prime}\right)+\frac{d^{2}}{d x^{2}}\left(\left(P_{4}-3 P_{6}^{\prime \prime}\right) \varphi^{\prime \prime}\right)+\frac{d}{d x}\left(\left(P_{2}-P_{4}^{\prime \prime}+3 P_{6}^{I V}\right) \varphi^{\prime}\right)+P_{0} \varphi=0
$$

To determine the boundary conditions for $u$, we consider the homogenous problem that is: put $f=0$ in (2.3) and using (2.4), we have

$$
\begin{aligned}
& \left\{\left(P_{6} u\right) \varphi^{V}-\left(\left(P_{6} u\right)^{\prime}-\left(P_{5} u\right)\right) \varphi^{I V}+\left(\left(P_{6} u\right)^{\prime \prime}-\left(P_{5} u\right)^{\prime}+\left(P_{4} u\right)\right) \varphi^{\prime \prime \prime}-\left(\left(P_{6} u\right)^{\prime \prime \prime}\right.\right. \\
& -\left(P_{5} u\right)^{\prime \prime}+\left(P_{4} u\right)^{\prime}-\left(P_{3} u\right) \varphi^{\prime \prime}+\left(\left(P_{6} u\right)^{I V}-\left(P_{5} u\right)^{\prime \prime \prime}+\left(P_{4} u\right)^{\prime \prime}-\left(P_{3} u\right)^{\prime}+\left(P_{2} u\right)\right) \varphi^{\prime} \\
& -\left(\left(P_{6} u\right)^{V}-\left(P_{5} u\right)^{I V}+\left(P_{4} u\right)^{\prime \prime \prime}-\left(P_{3} u\right)^{\prime \prime}+\left(P_{2} u\right)^{\prime}-\left(P_{1} u\right) \varphi\right\}_{a}^{b}=0
\end{aligned}
$$

But for the homogenous problem

$$
\varphi(a)=\varphi^{\prime}(a)=\varphi^{\prime \prime}(a)=\varphi(b)=\varphi^{\prime}(b)=\varphi^{\prime \prime}(b)=0
$$

Hence (2.7) becomes

$$
\begin{aligned}
& \left.P_{6} u\right|_{b} \varphi^{V}(b)-\left[\left(P_{6} u\right)^{\prime}-\left(P_{5} u\right)\right]_{b} \varphi^{N}(b)+\left[\left(P_{6} u\right)^{\prime \prime}-\left(P_{5} u\right)^{\prime}+\left(P_{4} u\right)\right]_{b} \varphi^{\prime \prime \prime}(b) \\
& -\left.P_{6} u\right|_{a} \varphi^{V}(a)+\left[\left(P_{6} u\right)^{\prime}-\left(P_{5} u\right)\right]_{a} \varphi^{I V}(a)-\left[\left(P_{6} u\right)^{\prime \prime}-\left(P_{5} u\right)^{\prime}+\left(P_{4} u\right)\right]_{a} \varphi^{\prime \prime \prime}(a)=0
\end{aligned}
$$

We choose the adjoint boundary conditions such that each of the coefficients of 
$\varphi^{V}(b), \varphi^{I V}(b), \varphi^{\prime \prime \prime}(b), \varphi^{V}(a), \varphi^{I V}(a)$ and $\quad \varphi^{\prime \prime \prime}(a)$

Vanish independently in (2.8), we get

$u(a)=0, u^{\prime}(a)=0, u^{\prime \prime}(a)=0, u(b)=0, u^{\prime}(b)=0, u^{\prime \prime}(b)=0$

To determine the solvability condition for the original problem and by using the relations (2.4), (1.2) and (2.9) in (2.3), we have

$$
\begin{aligned}
& P_{6} u^{\prime \prime \prime} \varphi^{\prime \prime}+\left(4 P_{6}^{\prime} u^{\prime \prime \prime}+P_{6} u^{I V}-P_{5} u^{\prime \prime \prime}\right) \varphi^{\prime}-\left(10 P_{6}^{\prime \prime} u^{\prime \prime \prime}+5 P_{6}^{\prime} u^{I V}+P_{6} u^{V}-4 P_{5}^{\prime} u^{\prime \prime \prime}-P_{5} u^{I V}+\right. \\
& \left.\left.+p_{4} u^{\prime \prime \prime}\right) \varphi\right\}_{a}^{b}=\int_{a}^{b} f u d x
\end{aligned}
$$

or

$\left[P_{6} u^{\prime \prime \prime} \beta_{6}+\left(4 P_{6}^{\prime} u^{\prime \prime \prime}+P_{6} u^{I V}-P_{5} u^{\prime \prime \prime}\right) \beta_{5}-\left(10 P_{6}^{\prime \prime} u^{\prime \prime \prime}+5 P_{6}^{\prime} u^{I V}+P_{6} u^{V}-4 P_{5}^{\prime} u^{\prime \prime \prime}\right.\right.$

$\left.\left.-P_{5} u^{I V}+P_{4} u^{\prime \prime \prime}\right) \beta_{4}\right]_{b}-\left[P_{6} u^{\prime \prime \prime} \beta_{3}+\left(4 P_{6}^{\prime} u^{\prime \prime \prime}+P_{6} u^{I V}-P_{5} u^{\prime \prime \prime}\right) \beta_{2}-\left(10 P_{6}^{\prime \prime} u^{\prime \prime \prime}\right.\right.$

$\left.\left.5 P_{6}^{\prime} u^{I V}+P_{6} u^{V}-4 P_{5}^{\prime} u^{\prime \prime \prime}-P_{5} u^{I V}+P_{4} u^{\prime \prime \prime}\right) \beta_{1}\right]_{a}=\int_{a}^{b} f(x) u(x) d x$

\section{(3)- Illustrative Example}

This example concerns the solvability condition of the following boundary problem :

$$
\begin{aligned}
& y^{V I}+56 \pi^{2} y^{I V}+784 \pi^{4} y^{\prime \prime}+2304 \pi^{6} y=\pi^{5} \cos 2 \pi x \\
& y(0)=\beta_{1}, y^{\prime}(0)=\beta_{2}, y^{\prime \prime}(0)=\beta_{3}, y(1)=\beta_{4}, y^{\prime}(1)=\beta_{5}, y^{\prime \prime}(1)=\beta_{6}
\end{aligned}
$$

Using the theorem we have

$$
p_{6}=1, p_{5}=0, p_{4}=56 \pi^{2}, p_{3}=0, p_{2}=784 \pi^{4}, p_{1}=0, p_{0}=2304 \pi^{6}
$$

The general solution of boundary value problem is $y=c_{1} \cos 2 \pi x+c_{2} \sin 2 \pi x+c_{3} \cos 4 \pi x+c_{4} \sin 4 \pi x+c_{5} \cos 6 \pi x+$

$$
+c_{6} \sin 6 \pi x+\frac{1}{1536} x \sin 2 \pi x
$$

The general solution of adjoint equation is $u=\frac{5}{3} \cos 2 \pi x-5 \sin 2 \pi x-\frac{8}{3} \cos 4 \pi x+\sin 4 \pi x+\cos 6 \pi x+\sin 6 \pi x$ and the adjoint boundary condition

$$
\begin{aligned}
& u(0)=u^{\prime}(0)=u^{\prime \prime}(0)=0, u^{\prime \prime \prime}(0)=-240 \pi^{3}, u^{I V}(0)=640 \pi^{4}, u^{V}(0)=8640 \pi^{5} \\
& u(1)=u^{\prime}(1)=u^{\prime \prime}(1)=0, \quad u^{\prime \prime \prime}(1)=-240 \pi^{3}, u^{I V}(1)=640 \pi^{4}, u^{V}(1)=8640 \pi^{5}
\end{aligned}
$$

Hence the solvability condition

$$
\begin{aligned}
& -240 \pi^{3} \beta_{6}+640 \beta_{5}-\left(8640 \pi^{5}+56 \pi^{2}(-240)\right) \beta_{4}-\left(-240 \pi^{3} \beta_{3}+640 \pi^{4} \beta_{2}-\right. \\
& -\left(8640 \pi^{5}+56 \pi^{2}\left(-240 \pi^{3}\right)\right) \beta_{1} \\
& \Rightarrow-240 \pi^{3}\left(\beta_{6}-\beta_{3}\right)+640 \pi^{4}\left(\beta_{5}-\beta_{2}\right)++4800 \pi^{5}\left(\beta_{4}-\beta_{1}\right)=\frac{5}{6} \pi^{5}
\end{aligned}
$$


if $\quad \beta_{6}=\beta_{3}, \beta_{4}=\beta_{1}, \beta_{5}-\beta_{2}=\frac{2 \pi}{1536}$

We have $\frac{5}{6} \pi^{5}=\frac{5}{6} \pi^{5}$

That is R.H.S =L.H.S

\section{(4)- Conclusions}

Through out the investigation of the solvability condition of sixth-order boundary value problem (1.1-1.2), we have seen that if the homogenous problem has non trivial solution, the corresponding nonhomogenous problem has a solution if the nonhomogenous term satisfied the condition (2.1).

\section{References}

1) Boyce, W. E. and Diprima, R. C. "Elementary differential equations and boundary value problems', John Wiley and Sons, Printed in U.S.A., 2004 .

2) Mahmood, A. H. "Solvability condition for certain eigenvalue problem by perturbation technique', Educ. Sci. J., Vol. (45), 2000.

3) Mahmood, A. H. and Jassim, E.A. 'Solvability condition for certain boundary value problem by perturbation method', Educ. Sci.J., Vol. (18), No.(2), P(90-103), 2006, In arabic .

4) Nayfeh, A. H. 'Introduction to perturbation technique', Mir Moscow, 1984.

5) Noor, M. A. and Mohyed-Din, S. T. "perturbation method for solving fourth order boundary value problems', Hindawi publishing corporation, Mathematical problem in engeineering. Vol. (2007).

6) Shen, Shaowei "Application of homotophy perturbation method to the fifth - order boundary value problems', Int. J. Contemp. math. Sci., Vol.(2), No.(25), p(1227-1236), 2007. 